N. TODA

KODAI MATH. J.

20 (1997), 189-207

\title{
ON THE FUNDAMENTAL INEQUALITY FOR NON-DEGENERATE HOLOMORPHIC CURVES
}

\author{
NoBUSHIge TODA
}

\section{Introduction}

Let

$$
f: C \longrightarrow P^{n}(\boldsymbol{C})
$$

be a holomorphic curve from $C$ into the $n$-dimensional complex projective space $P^{n}(\boldsymbol{C})$, where $n$ is a positive integer, and let

$$
\left(f_{1}, \ldots, f_{n+1}\right): \boldsymbol{C} \longrightarrow \boldsymbol{C}^{n+1}-\{\boldsymbol{0}\}
$$

be a reduced representation of $f$. We then write $f$ as follows:

$$
f=\left[f_{1}, \ldots, f_{n+1}\right] \text {. }
$$

We use the following notation:

$$
\|f(z)\|=\left(\left|f_{1}(z)\right|^{2}+\cdots+\left|f_{n+1}(z)\right|^{2}\right)^{1 / 2}
$$

and for a vector $\boldsymbol{a}=\left(a_{1}, \ldots, a_{n+1}\right)$ in $\boldsymbol{C}^{n+1}$

$$
\begin{gathered}
(\boldsymbol{a}, f)=a_{1} f_{1}+\cdots+a_{n+1} f_{n+1}, \\
(\boldsymbol{a}, f(z))=a_{1} f_{1}(z)+\cdots+a_{n+1} f_{n+1}(z), \\
\|\boldsymbol{a}\|=\left(\left|a_{1}\right|^{2}+\cdots+\left|a_{n+1}\right|^{2}\right)^{1 / 2} .
\end{gathered}
$$

The characteristic function $T(r, f)$ of $f$ is defined as follows (see [11]):

$$
T(r, f)=\frac{1}{2 \pi} \int_{0}^{2 \pi} \log \left\|f\left(r e^{i \theta}\right)\right\| d \theta-\log \|f(0)\| .
$$

Further, put

$$
U(z)=\max _{1 \leqq \jmath \leqq n+1}\left|f_{j}(z)\right|,
$$

then it is known $([1])$ that

This research was partially supported by Grant-in-Aid for Scientific Research (No. 08640194 and No. 09640180), Ministry of Education, Science and Culture.

Received July 5, 1996; revised August 28, 1997. 


$$
T(r, f)=\frac{1}{2 \pi} \int_{0}^{2 \pi} \log U\left(r e^{i \theta}\right) d \theta+O(1) .
$$

We suppose throughout the paper that $f$ is transcendental; that is to say,

$$
\lim _{r \rightarrow \infty} \frac{T(r, f)}{\log r}=\infty \text {. }
$$

We denote by $\rho(f)$ the order of $f$ :

$$
\rho(f)=\limsup _{r \rightarrow \infty} \frac{\log T(r, f)}{\log r}
$$

and by $S(r, f)$ any quantity satisfying

$$
S(r, f)=\left\{\begin{array}{lll}
O(\log r) & (r \rightarrow \infty) & \text { if } \rho(f)<\infty \\
O(\log r T(r, f)) & (r \rightarrow \infty, r \notin E) & \text { otherwise },
\end{array}\right.
$$

where $E$ is a subset of $[0, \infty)$ the measure of which is finite.

For meromorphic functions in $|z|<\infty$ we shall use the standard notation and symbols of the Nevanlinna theory of meromorphic functions ([3]).

For $\boldsymbol{a}=\left(a_{1}, \ldots, a_{n+1}\right) \in \boldsymbol{C}^{n+1}-\{0\}$ such that $(\boldsymbol{a}, f) \neq 0$, we write

$$
m(r, \boldsymbol{a}, f)=\frac{1}{2 \pi} \int_{0}^{2 \pi} \log \frac{\|\boldsymbol{a}\|\left\|f\left(r e^{i \theta}\right)\right\|}{\left|\left(\boldsymbol{a}, f\left(r e^{i \theta}\right)\right)\right|} d \theta
$$

and

Then we have

$$
N(r, \boldsymbol{a}, f)=N(r, 1 /(\boldsymbol{a}, f))
$$

$$
T(r, f)=N(r, \boldsymbol{a}, f)+m(r, \boldsymbol{a}, f)+O(1)
$$

It is called that the quantity

$$
\delta(\boldsymbol{a}, f)=1-\limsup _{r \rightarrow \infty} \frac{N(r, \boldsymbol{a}, f)}{T(r, f)}=\liminf _{r \rightarrow \infty} \frac{m(r, \boldsymbol{a}, f)}{T(r, f)}
$$

is the deficiency of $\boldsymbol{a}$ with respect to $f$. By (2)

$$
0 \leqq \delta(a, f) \leqq 1
$$

since $m(r, \boldsymbol{a}, f) \geqq 0$ and $N(r, \boldsymbol{a}, f) \geqq 0$ for $r \geqq 1$.

Further, let $\nu(c)$ be the order of zero of $(\boldsymbol{a}, f(z))$ at $z=c$ and for a positive integer $k$ let

$$
n_{k}(r, \boldsymbol{a}, f)=\sum_{|c| \leq r} \min \{\nu(c), k\} .
$$

Then, we put for $r>0$

$$
N_{k}(r, \boldsymbol{a}, f)=\int_{0}^{r} \frac{n_{\boldsymbol{k}}(t, \boldsymbol{a}, f)-n_{\boldsymbol{k}}(0, \boldsymbol{a}, f)}{t} d t+n_{k}(0, \boldsymbol{a}, f) \log r
$$

and put 


$$
\boldsymbol{\delta}_{k}(\boldsymbol{a}, f)=1-\limsup _{r \rightarrow \infty} \frac{N_{k}(r, \boldsymbol{a}, f)}{T(r, f)} .
$$

It is easy to see that $\delta(\boldsymbol{a}, f) \leqq \boldsymbol{\delta}_{k}(\boldsymbol{a}, f) \leqq 1$ by definition.

We say that $f$ is linearly non-degenerate (or simply, non-degenerate) if and only if $f_{1}, \ldots, f_{n+1}$ are linearly independent over $C$ and that $f$ is linearly degenerate when $f$ is not linearly non-degenerate.

It is well-known that $f$ is non-degenerate if and only if the Wronskian $W\left(f_{1}, \ldots, f_{n+1}\right)$ of $f_{1}, \ldots, f_{n+1}$ is not identically equal to zero. From now on we suppose that $f$ is non-degenerate.

Let $X$ be a subset of $\boldsymbol{C}^{n+1}-\{0\}$ such that $\# X \geqq n+1$. We suppose that $X$ is in general position; that is to say, any $n+1$ elements of $X$ are linearly independent. About 65 years ago, H. Cartan ([1]) proved the following fundamental inequality.

Theorem A. For any $\boldsymbol{a}_{1}, \ldots, \boldsymbol{a}_{q}$ in $X$,

$$
(q-n-1) T(r, f)<\sum_{j=1}^{q} N\left(r, \boldsymbol{a}_{\jmath}, f\right)-N\left(r, 1 / W\left(f_{1}, \ldots, f_{n+1}\right)\right)+S(r, f) .
$$

E. I. Nochka generalized this theorem to the case when $f$ is linearly degenerate (see [2], Chapter 3). Our first purpose of this paper is to give an improvement of Theorem $\mathrm{A}$.

Recently, we have introduced the following notion for holomorphic curves in [7], which corresponds to the derivative of meromorphic functions when $n=1$.

Definition A. We call the holomorphic curve induced by the mapping

$$
\left(f_{1}^{n+1}, \ldots, f_{n}^{n+1}, W\left(f_{1}, \ldots, f_{n+1}\right)\right): C \longrightarrow \boldsymbol{C}^{n+1}
$$

the derived holomorphic curve of $f$ and express it by $f^{*}$.

It is easy to see that $f^{*}$ is independent of the choice of reduced representation of $f([7])$.

Let $d(z)$ be an entire function such that the functions

$$
f_{\jmath}^{n+1} / d(j=1, \ldots, n) \text { and } W\left(f_{1}, \ldots, f_{n+1}\right) / d
$$

are entire functions without common zeros. Then,

$$
f^{*}=\left[f_{1}^{n+1} / d, \ldots, f_{n}^{n+1} / d, W\left(f_{1}, \ldots, f_{n+1}\right) / d\right] .
$$

We proved the following in [7].

THEOREM B. (a) $T\left(r, f^{*}\right) \leqq(n+1) T(r, f)-N(r, 1 / d)+S(r, f)$,

(b) $f^{*}$ is transcendental,

(c) $\rho\left(f^{*}\right)=\rho(f)$, 
(d) $f^{*}$ is not always non-degenerate.

Further, we introduced the following subset of $X$ in [8], which corresponds to the pole of meromorphic functions when $n=1$.

$$
X(0)=\left\{\boldsymbol{a}=\left(a_{1}, \ldots, a_{n+1}\right) \in X: a_{n+1}=0\right\} .
$$

It is easy to see that $\# X(0) \leqq n$ as $X$ is in general position.

Let $\boldsymbol{e}_{1}, \ldots, \boldsymbol{e}_{n+1}$ be the standard basis of $\boldsymbol{C}^{n+1}$. Then, we have

Theorem C. For any $\boldsymbol{a}_{1}, \ldots, \boldsymbol{a}_{q} \in X-X(0)(1 \leqq q<\infty)$,

$$
\sum_{j=1}^{q} m\left(r, \boldsymbol{a}_{\jmath}, f\right) \leqq m\left(r, \boldsymbol{e}_{n+1}, f^{*}\right)+S(r, f)
$$

(see Theorem 1 in [8] and [9]).

When $X(0)$ is empty, we can easily obtain Theorem A from Theorem B, (a) and Theorem $\mathrm{C}$, but Theorem $\mathrm{C}$ does not contain Theorem $\mathrm{A}$ when $X(0)$ is not empty. It is desirable for us to give a result which contains Theorem A. To that end, we shall introduce some new notions in Section 2, and in Section 3 we shall give a refinement of Theorem A and an improvement of the defect relation. In Section 4 we shall give an improvement of the second main theorem for moving targets obtained by M. Ru and W. Stoll $([4])$, which is the second purpose of this paper.

\section{Preliminaries and lemmas}

Let $f=\left[f_{1}, \ldots, f_{n+1}\right], T(r, f), X$ and $X(0)$ etc. be as in Section 1 .

DEFINITION 1. We put

$$
u(z)=\max _{1 \leq j \leq n}\left|f_{j}(z)\right|
$$

and

$$
t(r, f)=\frac{1}{2 \pi} \int_{0}^{2 \pi} \log u\left(r e^{i \theta}\right) d \theta-\frac{1}{2 \pi} \int_{0}^{2 \pi} \log u\left(e^{i \theta}\right) d \theta .
$$

It is easy to see the following properties of $t(r, f)$.

PROPOSITION 1. (a) $t(r, f)$ is independent of the choice of reduced representation of $f$.

(b) $t(r, f) \leqq T(r, f)+O(1)$.

(c) $N\left(r, 1 / f_{j}\right) \leqq t(r, f)+O(1)(j=1, \ldots, n)$.

As an improvement of Theorem B, (a) (=Lemma 3([7])), we can prove the following 
LEMMA 1. $T\left(r, f^{*}\right) \leqq T(r, f)+n t(r, f)-N(r, 1 / d)+S(r, f)$.

Proof. From the inequality

$$
\begin{aligned}
\left\|f^{*}(z)\right\|^{2}= & \left\{\left|f_{1}(z)\right|^{2(n+1)}+\cdots+\left|f_{n}(z)\right|^{2(n+1)}+\left|W\left(f_{1}, \ldots, f_{n+1}\right)(z)\right|^{2}\right\} /|d(z)|^{2} \\
\leqq & \frac{U(z)^{2}}{|d(z)|^{2}}\left\{\left|f_{1}(z)\right|^{2 n}+\cdots+\left|f_{n}(z)\right|^{2 n}\right. \\
& \left.\quad+\left|f_{1}(z) \cdots f_{n}(z)\right|^{2} \frac{\left|W\left(f_{1}, \ldots, f_{n+1}\right)(z)\right|^{2}}{\left|f_{1}(z) \cdots f_{n+1}(z)\right|^{2}}\right\} \\
\leqq & \frac{U(z)^{2}}{|d(z)|^{2}} u(z)^{2 n}\left\{n+\frac{\left|W\left(f_{1}, \ldots, f_{n+1}\right)(z)\right|^{2}}{\left|f_{1}(z) \cdots f_{n+1}(z)\right|^{2}}\right\}
\end{aligned}
$$

and from the fact that

$$
\frac{1}{2 \pi} \int_{0}^{2 \pi} \log ^{+} \frac{\left|W\left(f_{1}, \ldots, f_{n+1}\right)\left(r e^{i \theta}\right)\right|}{\left|f_{1}\left(r e^{i \theta}\right) \cdots f_{n+1}\left(r e^{i \theta}\right)\right|} d \theta=S(r, f)
$$

(see [1], p. 12-p. 15), we easily obtain our lemma by (1).

DEFINITION 2. We put

$$
\omega=\liminf _{r \rightarrow \infty} \frac{t(r, f)}{T(r, f)} \text { and } \Omega=\limsup _{r \rightarrow \infty} \frac{t(r, f)}{T(r, f)} .
$$

PROPOSITION 2. $0 \leqq \omega \leqq \Omega \leqq 1$. Put

Suppose now that $X(0)$ is not empty and that $X(0)=\left\{\boldsymbol{b}_{1}, \ldots, \boldsymbol{b}_{\nu}\right\}(1 \leqq \nu \leqq n)$.

$$
\left(\boldsymbol{b}_{j}, f\right)=G, \quad(j=1, \ldots, \nu) .
$$

DEFINITION 3. We express the holomorphic curve induced by the mapping

$$
\left(G_{1} \cdots G_{\nu} f_{1}^{n+1-\nu}, \ldots, G_{1} \cdots G_{\nu} f_{n}^{n+1-\nu}, W\left(f_{1}, \ldots, f_{n+1}\right)\right): \boldsymbol{C} \longrightarrow \boldsymbol{C}^{n+1}
$$

by $f_{2}^{*}$.

It is easy to see that $f_{\nu}^{*}$ is independent of the choice of reduced representation of $f$ as in the case of $f^{*}$.

Let $d_{\nu}$ be an entire function such that the functions

$$
G_{1} \ldots G_{\nu} f_{\jmath}^{n+1-\nu} / d_{\nu}(j=1, \ldots, n) \text { and } W\left(f_{1}, \ldots, f_{n+1}\right) / d_{\nu}
$$

are entire functions without common zeros. Then we have the following

LEMMA 2.

$$
T\left(r, f_{\nu}^{*}\right) \leqq T(r, f)+(n-\nu) t(r, f)+\sum_{j=1}^{\nu} N\left(r, 1 / G_{j}\right)-N\left(r, 1 / d_{\nu}\right)+S(r, f) .
$$


Proof. We suppose without loss of generality that $\boldsymbol{b}_{1}, \ldots, \boldsymbol{b}_{\nu}, \boldsymbol{e}_{\nu+1}, \ldots, \boldsymbol{e}_{n}$, $\boldsymbol{e}_{n+1}$ are linearly independent because $\boldsymbol{b}_{1}, \ldots, \boldsymbol{b}_{\nu}$ are linearly independent vectors in $X(0)$.

Now, put $\Pi=G_{1} \cdots G_{\nu}$ and $l=n-\nu$. Then, we have the inequality

$$
\begin{aligned}
& \left\|f_{\nu}^{*}(z)\right\|^{2} \\
& =\left\{|\Pi(z)|^{2}\left|f_{1}(z)\right|^{2(l+1)}+\cdots+|\Pi(z)|^{2}\left|f_{n}(z)\right|^{2(l+1)}+|W(z)|^{2}\right\} /\left|d_{\nu}(z)\right|^{2} \\
& =\frac{|\Pi(z)|^{2}}{\left|d_{\nu}(z)\right|^{2}}\left\{\left|f_{1}(z)\right|^{2(l+1)}+\cdots+\left|f_{n}(z)\right|^{2(l+1)}+\frac{\left|f_{\nu+1}(z) \cdots f_{n+1}(z) W(z)\right|^{2}}{\left|\Pi(z) f_{\nu+1}(z) \cdots f_{n+1}(z)\right|^{2}}\right\} \\
& \leqq \frac{|\Pi(z)|^{2}}{\left|d_{\nu}(z)\right|^{2}} U(z)^{2} u(z)^{2(n-\nu)}\left(n+\frac{|W(z)|^{2}}{\left|\Pi(z) f_{\nu+1}(z) \cdots f_{n+1}(z)\right|^{2}}\right),
\end{aligned}
$$

where $W=W\left(f_{1}, \ldots, f_{n+1}\right)$. From this inequality we easily obtain our lemme as in the proof of Lemma 1 since

$$
W\left(f_{1}, \ldots, f_{n+1}\right)=c_{\nu} W\left(G_{1}, \ldots, G_{\nu}, f_{\nu+1}, \ldots, f_{n+1}\right) \quad\left(c_{\nu} \neq 0 \text {, constant }\right) .
$$

As in the case of (b) and (c) of Theorem B, we can prove the following properties of $f_{2}^{*}$.

Proposition 3. (a) $f_{\nu}^{*}$ is transcendental,

(b) $\rho\left(f_{\nu}^{*}\right)=\rho(f)$.

To prove this proposition we use the relation

$$
\begin{aligned}
\frac{W\left(f_{1}, \ldots, f_{n+1}\right)}{G_{1} \cdots G_{\nu} f_{1}^{n+1-\nu}} & =\frac{f_{1}}{G_{1}} \cdots \frac{f_{1}}{G_{\nu}} \cdot \frac{W\left(f_{1}, \ldots, f_{n+1}\right)}{f_{1}^{n+1}} \\
& =\frac{f_{1}}{G_{1}} \cdots \frac{f_{1}}{G_{\nu}} \cdot W\left(\left(\frac{f_{2}}{f_{1}}\right)^{\prime}, \ldots,\left(\frac{f_{n+1}}{f_{1}}\right)^{\prime}\right)
\end{aligned}
$$

and the fact that for $G=a_{1} f_{1}+\cdots+a_{n} f_{n}$

$$
\frac{G}{f_{1}}=a_{1}+\sum_{j=2}^{n} a_{j} \cdot \frac{f_{j}}{f_{1}} \text { is }\left\{\begin{array}{l}
\text { rational if so are } f_{j} / f_{1}(j=2, \ldots, n) ; \\
\text { of order }<\rho(f) \text { if } \rho\left(f_{j} / f_{1}\right)<\rho(f)(j=2, \ldots, n) .
\end{array}\right.
$$

\section{Fundamental inequality}

Let $f=\left[f_{1}, \ldots, f_{n+1}\right], T(r, f), X$ and $X(0)$ etc. be as in Section 1. Suppose that $X(0)$ is not empty and that

We put

$$
X(0)=\left\{\boldsymbol{b}_{1}, \ldots, \boldsymbol{b}_{\nu}\right\} \quad(1 \leqq \nu \leqq n) .
$$

$$
\left(\boldsymbol{b}_{j}, f\right)=G, \quad(j=1, \ldots, \nu) .
$$

We suppose without loss of generality that $\boldsymbol{b}_{1}, \ldots, \boldsymbol{b}_{\nu}, \boldsymbol{e}_{\nu+1}, \ldots, \boldsymbol{e}_{n}, \boldsymbol{e}_{n+1}$ are 
linearly independent as in the proof of Lemma 2.

THEOREM 1. For any $\boldsymbol{a}_{1}, \ldots, \boldsymbol{a}_{q} \in X-X(0)(1 \leqq q<\infty)$,

$$
\begin{aligned}
& \sum_{\jmath=1}^{q} m\left(r, \boldsymbol{a}_{\jmath}, f\right) \leqq m\left(r, \boldsymbol{e}_{n+1}, f_{\nu}^{*}\right)+S(r, f) \\
& \quad \leqq T(r, f)+(n-\nu) t(r, f)+\sum_{j=1}^{\nu} N\left(r, 1 / G_{j}\right)-N(r, 1 / W)+S(r, f) .
\end{aligned}
$$

where $W=W\left(f_{1}, \ldots, f_{n+1}\right)$.

Proof. We have only to prove this theorem for $q \geqq n+1$. Put

$$
\left(\boldsymbol{a}_{\jmath}, f\right)=F_{\jmath} \quad(j=1, \ldots, q) .
$$

For any $z(\neq 0)$ arbitrarily fixed, let

$$
\left|F_{\jmath_{1}}(z)\right| \leqq\left|F_{\jmath_{2}}(z)\right| \leqq \cdots \leqq\left|F_{\jmath_{q}}(z)\right| \quad\left(1 \leqq j_{1}, \ldots, j_{q} \leqq q\right) .
$$

Then there is a positive constant $K$ such that

$$
\begin{array}{ll}
\|f(z)\| \leqq K\left|F_{\jmath_{p}}(z)\right| & (p=n+1, \ldots, q), \\
\left|F_{\jmath_{p}}(z)\right| \leqq K\|f(z)\| & (p=1, \ldots, q)
\end{array}
$$

and since the $n+1$-th elements of vectors $a$, are different from zero,

$$
\left|f_{n+1}(z)\right| \leqq K\left\{u(z)+\left|F_{\jmath_{p}}(z)\right|\right\} \quad(p=1, \ldots, q) .
$$

(From now on we denote by $K$ a positive constant, which may be different from each other when it appears in different places.)

(i) The case when $u(z) \leqq\left|F_{\jmath_{1}}(z)\right|$.

Since $\|f(z)\| \leqq K\left|F_{\jmath_{1}}(z)\right|$ in this case by (5), we have

$$
\prod_{j=1}^{q} \frac{\left\|\boldsymbol{a}_{j}\right\|\|f(z)\|}{\left|\left(\boldsymbol{a}_{\jmath}, f(z)\right)\right|} \leqq K .
$$

(ii) The case when $\left|F_{\jmath_{1}}(z)\right|<u(z)$.

In this case, by using (5) we have

and by (3) we obtain

$$
\|f(z)\| \leqq K u(z)
$$

(7) $\quad \prod_{\jmath=1}^{q} \frac{\left\|\boldsymbol{a}_{j}\right\|\|f(\boldsymbol{z})\|}{\left|\left(\boldsymbol{a}_{\jmath}, f(z)\right)\right|} \leqq K \prod_{p=1}^{n} \frac{u(z)}{\left|F_{\jmath_{p}}(z)\right|}=K \frac{\left|G_{1}(z) \cdots G_{\nu}(z)\right| u(z)^{n}}{\left|G_{1}(z) \cdots G_{\nu}(z) F_{\jmath_{1}}(z) \cdots F_{\jmath_{n}}(z)\right|}=(*)$.

Here, we put

and let

$$
G_{\imath}=H_{\imath}(i=1, \ldots, \nu), \quad F_{\jmath_{p}}=H_{\nu+p}(p=1, \ldots, n)
$$


196

NOBUSHIGE TODA

$$
\left|H_{\imath_{1}}(z)\right| \leqq\left|H_{\imath_{2}}(z)\right| \leqq \cdots \leqq\left|H_{\imath_{n+\nu}}(z)\right|
$$

Then for $k=2, \ldots, \nu$

$$
u(z) \leqq K\left|H_{\imath_{n+k}}(z)\right|
$$

and we have for $W=W\left(f_{1}, \ldots, f_{n+1}\right)$ and $\Pi=G_{1} \cdots G_{\nu}$

$$
\begin{aligned}
(*) & \leqq K \frac{|\Pi(z)| u(z)^{n+1-\nu}}{\left|H_{\imath_{1}}(z) \cdots H_{\imath_{n+1}}(z)\right|} \\
& =K \frac{|\Pi(z)| u(z)^{n+1-\nu}}{|W(z)|} \cdot \frac{|W(z)|}{\left|H_{\imath_{1}}(z) \cdots H_{\imath_{n+1}}(z)\right|} \\
& =K \frac{|\Pi(z)| u(z)^{n+1-\nu}}{|W(z)|} \cdot \frac{\left|W\left(H_{\imath_{1}}, \cdots, H_{\imath_{n+1}}\right)(z)\right|}{\left|H_{\imath_{1}}(z) \cdots H_{\imath_{n+1}}(z)\right|}
\end{aligned}
$$

since $H_{\imath_{1}}, \ldots, H_{\imath_{n+1}}$ are linearly independent and

$$
W\left(H_{\imath_{1}}, \ldots, H_{\imath_{n+1}}\right)=c W\left(f_{1}, \ldots, f_{n+1}\right) \quad(c \neq 0 \text {, constant }) .
$$

From (6), (7) and ( $\left.7^{\prime}\right)$ we obtain the inequality

$$
\begin{aligned}
& \sum_{j=1}^{q} \log \frac{\left\|\boldsymbol{a}_{j}\right\|\|f(z)\|}{\left|\left(\boldsymbol{a}_{j}, f(z)\right)\right|} \leqq \log ^{+} \frac{|\Pi(z)| u(z)^{n+1-\nu}}{|W(z)|} \\
&+\sum_{\left(o_{1}, \ldots, j_{n+1}\right)} \log ^{+} \frac{\left|W\left(H_{\imath_{1}}, \ldots, H_{\imath_{n+1}}\right)(z)\right|}{\left|H_{\imath_{1}}(z) \cdots H_{\imath_{n+1}}(z)\right|}+\log ^{+} K,
\end{aligned}
$$

where $\sum_{\left(j_{1}, \ldots, j_{n+1}\right)}$ is the summation taken over all combinations $\left(j_{1}, \ldots, j_{n+1}\right)$ chosen from $\{1, \ldots, q\}$ which appear in the above argument when we vary $z$ in $0<|z|<\infty$, and integrating this inequality from 0 to $2 \pi$ with respect to $\theta$, where $z=r e^{i \theta}$, we obtain the inequality

$$
\sum_{j=1}^{q} m\left(r, \boldsymbol{a}_{\jmath}, f\right) \leqq m\left(r, \boldsymbol{e}_{n+1}, f_{\nu}^{*}\right)+S(r, f)=(* *)
$$

since, by applying (1) to $f_{\nu}^{*}$ and to the following equality

$$
\begin{aligned}
\log ^{+} & \frac{|\Pi(z)| u(z)^{n+1-\nu}}{|W(z)|}=\log \max \left\{\frac{|\Pi(z)| u(z)^{n+1-\nu}}{\left|d_{\nu}(z)\right|}, \frac{|W(z)|}{\left|d_{\nu}(z)\right|}\right\}-\log \frac{|W(z)|}{\left|d_{\nu}(z)\right|} \\
= & \log \frac{1}{\left|d_{\nu}(z)\right|} \max \left\{|\Pi(z)|\left|f_{1}(z)\right|^{n+1-\nu}, \ldots,|\Pi(z)|\left|f_{n}(z)\right|^{n+1-\nu},|W(z)|\right\} \\
& -\log \frac{|W(z)|}{\left|d_{\nu}(z)\right|}
\end{aligned}
$$

we have

$$
\begin{aligned}
\frac{1}{2 \pi} \int_{0}^{2 \pi} \log ^{+} \frac{\left|\Pi\left(r e^{i \theta}\right)\right| u\left(r e^{i \theta}\right)^{n+1-\nu}}{\left|W\left(r e^{i \theta}\right)\right|} d \theta & =T\left(r, f_{\nu}^{*}\right)-N\left(r, 1 /\left(W / d_{\nu}\right)\right)+O(1) \\
& =m\left(r, \boldsymbol{e}_{n+1}, f_{\nu}^{*}\right)+O(1)
\end{aligned}
$$


by using $N\left(r, e_{n+1}, f_{\nu}^{*}\right)=N\left(r, 1 /\left(W / d_{\nu}\right)\right)$ and we have for each $\left(j_{1}, \ldots, j_{n+1}\right)$

$$
\frac{1}{2 \pi} \int_{0}^{2 \pi} \log ^{+} \frac{\left|W\left(H_{\imath_{1}}, \ldots, H_{\imath_{n+1}}\right)\left(r e^{i \theta}\right)\right|}{\left|H_{\imath_{1}}\left(r e^{i \theta}\right) \cdots H_{\imath_{n+1}}\left(r e^{i \theta}\right)\right|} d \theta=S(r, f)
$$

as in the proof of Lemma 1 (see [1], p. 12-p. 15), and by Lemma 2

$$
(* *) \leqq T(r, f)+(n-\nu) t(r, f)+\sum_{j=1}^{\nu} N\left(r, 1 / G_{j}\right)-N(r, 1 / W)+S(r, f) .
$$

THEOREM 2. Let $\boldsymbol{a}_{1}, \ldots, \boldsymbol{a}_{q}$ be any vectors of $X$ such that the number of elements of the set $X(0) \cap\left\{\boldsymbol{a}_{1}, \ldots, \boldsymbol{a}_{q}\right\}$ is equal to $\mu(0 \leqq \mu \leqq n)$. Then, we have

$$
\sum_{j=1}^{q} m\left(r, \boldsymbol{a}_{j}, f\right) \leqq(\mu+1) T(r, f)+(n-\mu) t(r, f)-N(r, 1 / W)+S(r, f),
$$

where $W=W\left(f_{1}, \ldots, f_{n+1}\right)$.

Proof. We easily obtain this theorem from Theorem $\mathrm{C}$ and Lemma 1 for $\mu=0$. When $1 \leqq \mu \leqq n$, put

and

$$
X(0) \cap\left\{a_{1}, \ldots, \boldsymbol{a}_{q}\right\}=\left\{\boldsymbol{a}_{1}, \ldots, \boldsymbol{a}_{\mu}\right\}
$$

$$
\left(\boldsymbol{a}_{\jmath}, f\right)=G_{\jmath} \quad(j=1, \ldots, \mu) .
$$

Then, from Theorem 1 we have

$$
\sum_{j=\mu+1}^{q} m\left(r, \boldsymbol{a}_{\jmath}, f\right) \leqq T(r, f)+(n-\mu) t(r, f)+\sum_{j=1}^{\mu} N\left(r, 1 / G_{j}\right)-N(r, 1 / W)+S(r, f) .
$$

Adding $\sum_{j=1}^{\mu} m(r, \boldsymbol{a}, f)$ to both sides of this inequality we have this theorem as

$$
m\left(r, \boldsymbol{a}_{j}, f\right)+N\left(r, 1 / G_{j}\right)=T(r, f)+O(1) \quad(j=1, \ldots, \mu) .
$$

Remark 1. $(\mu+1) T(r, f)+(n-\mu) t(r, f) \leqq(n+1) T(r, f)+O(1)$ since $t(r, f) \leqq T(r, f)+O(1)$, and so Theorem 2 is an improvement of Theorem A.

COROLlaRY 1 (Defect relation). Under the same circumstances as in Theorem 2,

$$
\sum_{j=1}^{q} \delta_{n}\left(\boldsymbol{a}_{\jmath}, f\right) \leqq \mu+1+(n-\mu) \Omega .
$$

In fact, from Theorem 2 we obtain the inequality

$$
\begin{aligned}
(q-\mu-1) T(r, f) & \leqq \sum_{j=1}^{q} N\left(r, \boldsymbol{a}_{\jmath}, f\right)+(n-\mu) t(r, f)-N(r, 1 / W)+S(r, f) \\
& \leqq \sum_{j=1}^{q} N_{n}(r, \boldsymbol{a}, f)+(n-\mu) t(r, f)+S(r, f)
\end{aligned}
$$

by (2) for the first inequality and by the method used in [1], p. 14 for the 
second inequality, which reduces to our corollary as usual.

Remark 2. (i) $\mu+1+(n-\mu) \Omega \leqq n+1$ and the equality holds if and only if $\mu=n$ or $\Omega=1$.

(ii) If $\rho(f)$ is finite, we can change $\Omega$ to $\omega$ in Corollary 1 .

The number " $\mu+1+(n-\mu) \Omega$ " increases with $\mu(0 \leqq \mu \leqq n)$ when $\Omega<1$. If $\mu$ increases to $n$ when $q$ tends to $\infty$, the bound " $\mu+1+(n-\mu) \Omega$ " of this corollary increases to $n+1$ for any $\Omega<1$. There exist, however, examples of $X$ for which $\mu$ does not increase to $n$ even when $q$ tends to $\infty$ and examples of holomorphic curves with $\Omega<1$ and, by using the following notion introduced in [10], we obtain a refinement of the defect relation as follows.

Definition B ([10], Definition 1$)$. We say that

(i) $X$ is maximal (in the sense of general position) if and only if for any $Y$ in general position such that $X \subset Y \subset C^{n+1}, X=Y$.

(ii) $X$ is $\nu$-maximal if $X$ is maximal and $\# X(0)=\nu$.

Proposition 4. For any $\nu(1 \leqq \nu \leqq n)$, there is a $\nu$-maximal subset of $\boldsymbol{C}^{n+1}$ in the sense of general position ([10], Theorem 1).

COROLlary 2 (Defect relation). Let $X$ be a $\nu$-maximal subset of $\boldsymbol{C}^{n+1}$ in the sense of general position. Then, we have

$$
\sum_{a \in X} \delta_{n}(a, f) \leqq \nu+1+(n-\nu) \Omega .
$$

In fact, when $\#\left\{\boldsymbol{a} \in X: \boldsymbol{\delta}_{n}(\boldsymbol{a}, f)>0\right\}<\infty$, there is nothing to prove by

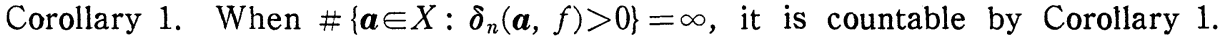
Let

$$
\left\{\boldsymbol{a} \in X: \delta_{n}(\boldsymbol{a}, f)>0\right\}=\left\{\boldsymbol{a}_{1}, \boldsymbol{a}_{2}, \ldots\right\},
$$

and without loss of generality we put

$$
X(0) \cap\left\{\boldsymbol{a}_{1}, \boldsymbol{a}_{2}, \ldots\right\}=\left\{\boldsymbol{a}_{1}, \ldots, \boldsymbol{a}_{p}\right\} \quad(0 \leqq p \leqq \nu) .
$$

Then, by Corollary 1 , for any $q>0$

$$
\sum_{j=1}^{q} \boldsymbol{\delta}_{n}\left(\boldsymbol{a}_{\jmath}, f\right) \leqq p+1+(n-p) \Omega \leqq \nu+1+(n-\nu) \Omega
$$

and letting $q$ tend to $\infty$ we have

$$
\sum_{\boldsymbol{a} \in X} \boldsymbol{\delta}_{n}(\boldsymbol{a}, f)=\sum_{\jmath=1}^{\infty} \boldsymbol{\delta}_{n}\left(\boldsymbol{a}_{\jmath}, f\right) \leqq \nu+1+(n-\nu) \Omega
$$

since $\nu$ is independent of $q$.

We here give some examples of $f$ for which $\Omega<1$. Let $a_{\jmath}(j=1, \ldots, n)$ be real numbers satisfying $0<a_{1}<a_{2}<\cdots<a_{n-1}<a_{n}$. 
Example 1. A holomorphic curve for which $\Omega<1$.

We consider the following holomorphic curve

$$
f=\left[1, e^{a} 1^{z}, e^{a 2^{2}}, \ldots, e^{a n^{2}}\right] .
$$

Then, for $z=r e^{i \theta}(r>0)$,

$$
U(z)=\left\{\begin{array}{cl}
1 & \left(\frac{\pi}{2} \leqq \theta \leqq \frac{3}{2} \pi\right) \\
\exp \left(r a_{n} \cos \theta\right) & \left(0 \leqq \theta<\frac{\pi}{2}, \frac{3}{2} \pi<\theta \leqq 2 \pi\right)
\end{array}\right.
$$

and by (1) we have

$$
T(r, f)=\frac{a_{n}}{\pi} r+O(1)
$$

On the other hand

$$
u(z)=\left\{\begin{array}{cl}
1 & \left(\frac{\pi}{2} \leqq \theta \leqq \frac{3}{2} \pi\right) \\
\exp \left(r a_{n-1} \cos \theta\right) & \left(0 \leqq \theta<\frac{\pi}{2}, \frac{3}{2} \pi<\theta \leqq 2 \pi\right)
\end{array}\right.
$$

and we have

$$
t(r, f)=\frac{a_{n-1}}{\pi} r+O(1) .
$$

We have $\omega=\Omega=a_{n-1} / a_{n}$, which is smaller than 1 .

Example 2. A holomorphic curve for which $\Omega=0$.

We consider the following holomorphic curve

$$
f=\left[1, e^{a_{1} z}, \ldots, e^{a n-1^{z}}, e^{z^{2}}\right] .
$$

Then, by a simple calculation we have

$$
T(r, f) \geqq \frac{r^{2}}{4 \pi}+O(1)
$$

and $t(r, f)$ is the same as that given in Example 1 , so that $\Omega=0$.

\section{Extension of the second fundamental theorem}

Let $f=\left[f_{1}, \ldots, f_{n_{+1}}\right],\|f(z)\|, T(r, f)$ and $U(z)$ be as in Section 1 . We set

$$
\Gamma=\left\{a: \text { meromorphic in }|z|<\infty, T(r, a)=S_{0}(r, f)\right\},
$$

where $S_{0}(r, f)$ is any quantity satisfying

$$
S_{0}(r, f)=o(T(r, f)) \quad(r \rightarrow \infty) .
$$


Note that $\Gamma$ is a field. Further we set

$$
\Gamma^{+}=\left\{\beta\left(\left|a_{1}\right|+\left|a_{2}\right|+\cdots+\left|a_{m}\right|\right)^{k}: a_{j} \in \Gamma ; \beta>0 \text {, constant; } m, k \in \boldsymbol{N}\right\},
$$

where $N$ is the set of positive integers. Observe that

$$
\begin{gathered}
\frac{1}{2 \pi} \int_{0}^{2 \pi} \log ^{+}\left(\left|a_{1}\left(r e^{i \theta}\right)\right|+\cdots+\left|a_{m}\left(r e^{i \theta}\right)\right|\right) d \theta \leqq \sum_{j=1}^{m} m\left(r, a_{\jmath}\right)+O(1) \\
\leqq \sum_{j=1}^{m} T\left(r, a_{j}\right)+O(1)=S_{0}(r, f) .
\end{gathered}
$$

From now on, we use $K(z)$ as a representative for any functions of $\Gamma^{+}$for brevity, and so $K(z)$ may be different from each other when it appears in different places. Note that

$$
\int_{0}^{2 \pi} \log ^{+} K\left(r e^{i \theta}\right) d \theta=S_{0}(r, f) .
$$

From now on throughout the section we suppose that $f$ is linearly nondegenerate over $\Gamma$. Let

$$
\boldsymbol{S}_{0}(f)=\left\{\boldsymbol{A}=\left[a_{1}, \ldots, a_{n+1}\right]: \begin{array}{l}
\text { holomorphic curve from } \boldsymbol{C} \text { into } P^{n}(\boldsymbol{C}), \\
T(r, \boldsymbol{A})=S_{0}(r, f)
\end{array}\right\}
$$

and let $X$ be a subset of $S_{0}(f)$. We suppose that $\# X \geqq n+1$ and $X$ is in general position; that is to say, for any $n+1$ elements

$$
\boldsymbol{A}_{\jmath}=\left[a_{1,}, \ldots, a_{n+1,}\right] \quad(j=1, \ldots, n+1)
$$

of $X, \operatorname{det}\left(a_{\imath \jmath}\right)$ is not identically equal to zero (see [10], $\S 4$ ). This is independent of the choice of reduced representations of $\boldsymbol{A}_{j} \in X$. It is clear that

Put

$$
S_{0}(f) \supset P^{n}(C) .
$$

$$
X(0)=\left\{\boldsymbol{A}=\left[a_{1}, \ldots, a_{n+1}\right] \in X: a_{n+1}=0\right\} .
$$

Definition C ([10], Definition 2). We say that $X$ is $\nu$-maximal in the sense of general position if and only if it satisfies the following conditions (i) and (ii):

(i) $X$ is maximal in the sense of general position; that is to say, for any subset $Y$ of $\boldsymbol{S}_{0}(f)$ in general position such that $X \subset Y \subset \boldsymbol{S}_{0}(f), X=Y$;

(ii) $\# X(0)=\nu$.

Remark 3. $0 \leqq \nu \leqq n$.

Proposition 5. For any $\nu(1 \leqq \nu \leqq n)$, there is a v-maximal subset of $\boldsymbol{S}_{0}(f)$ in the sense of general position ([10], Theorem 2).

We use the following notation in this section. For any $\boldsymbol{A}=\left[a_{1}, \ldots, a_{n+1}\right]$ 
of $S_{0}(f)$, we set

and

$$
(\boldsymbol{A}, f)=a_{1} f_{1}+\cdots+a_{n+1} f_{n+1}
$$

$$
(\boldsymbol{A}, f)(z)=a_{1}(z) f_{1}(z)+\cdots+a_{n+1}(z) f_{n+1}(z)
$$

Then we have the following (see [8], Proposition 2):

LEMMA 3. (a) $a_{\imath} / a_{j} \in \Gamma$ if $\quad a, \neq 0$.

(b) $(\boldsymbol{A}, f) \neq 0$.

We put for $A$ of $X$

and

$$
\begin{gathered}
m(r, \boldsymbol{A}, f)=\frac{1}{2 \pi} \int_{0}^{2 \pi} \log \frac{\left\|\boldsymbol{A}\left(r e^{i \theta}\right)\right\|\left\|f\left(r e^{i \theta}\right)\right\|}{\left|(\boldsymbol{A}, f)\left(r e^{i \theta}\right)\right|} d \theta \\
N(r, \boldsymbol{A}, f)=N(r, 1 /(\boldsymbol{A}, f))
\end{gathered}
$$

$$
\delta(\boldsymbol{A}, F)=\liminf _{r \rightarrow \infty} \frac{m(r, \boldsymbol{A}, f)}{T(r, f)} .
$$

Proposition 6. (a) $m(r, \boldsymbol{A}, f)+N(r, \boldsymbol{A}, f)=T(r, f)+S_{0}(r, f)$.

(b) $0 \leqq \delta(\boldsymbol{A}, f)=1-\limsup _{r \rightarrow \infty} \frac{N(r, \boldsymbol{A}, f)}{T(r, f)} \leqq 1$.

These are trivial by definition.

For any $\boldsymbol{A}=\left[a_{1}, \ldots, a_{n+1}\right]$ and $\boldsymbol{B}=\left[b_{1}, \ldots, b_{n+1}\right]$ of $\boldsymbol{S}_{0}(f)$ such that $a_{3} \neq 0$, $b_{k} \neq 0$, put $(\boldsymbol{A}, f)=F$ and $(\boldsymbol{B}, f)=G$. Then, we have the following lemma.

$$
\text { LEMMA 4. } T\left(r, \frac{F / a_{\jmath}}{G / b_{k}}\right) \leqq 2 n T(r, f)+S_{0}(r, f) . \quad \text { ([8], Lemma 6) }
$$

For $\boldsymbol{A}=\left[a_{1}, \ldots, a_{n+1}\right]$ of $X$, let $a_{\jmath_{0}}$ be the first element not identically equal to zero. Then, we put

$$
\begin{gathered}
\tilde{\boldsymbol{A}}=\left(a_{1} / a_{\jmath_{0}}, \ldots, a_{n+1} / a_{\jmath_{0}}\right)=\left(g_{1}, \ldots, g_{n+1}\right), \\
\tilde{X}=\{\tilde{\boldsymbol{A}}: \boldsymbol{A} \in X\}, \quad \tilde{X}(0)=\{\tilde{\boldsymbol{A}}: \boldsymbol{A} \in X(0)\}, \quad\|\tilde{\boldsymbol{A}}(z)\|=\|\boldsymbol{A}(z)\| /\left|a_{\jmath_{0}}(z)\right|
\end{gathered}
$$

and for $(\boldsymbol{A}, f)=F$

$$
\frac{(\boldsymbol{A}, f)}{a_{\jmath_{0}}}=(\tilde{\boldsymbol{A}}, f)=\tilde{F}=\sum_{\jmath=1}^{n+1} g_{\jmath} f_{\jmath} .
$$

Then, it is clear that $\tilde{X}$ is in general position; that is to say, for any $n+1$ elements

of $\tilde{X}$,

$$
\tilde{\boldsymbol{A}}_{\imath}=\left(g_{i 1}, \ldots, g_{\imath n+1}\right) \quad(i=1, \ldots, n+1)
$$

$$
\operatorname{det}\left(g_{\imath}\right) \neq 0
$$


and $g_{j} \in \Gamma$ by Lemma 3 , (a).

Let $f$ and $X$ be those given above in this section. Then, we have the following extension of the second fundamental theorem.

Theorem 3. Let $\boldsymbol{A}_{1}, \ldots, \boldsymbol{A}_{q}$ be any elements in $X-X(0)(1 \leqq q<\infty)$ and let $\boldsymbol{B}_{1}, \ldots, \boldsymbol{B}_{\mu}$ be in $X(0)(0 \leqq \mu \leqq n)$. Then, for any positive number $\varepsilon$,

$$
\sum_{\jmath=1}^{q} m\left(r, \boldsymbol{A}_{\jmath}, f\right) \leqq(1+\varepsilon) T(r, f)+(n-\mu) t(r, f)+\sum_{l=1}^{\mu} N\left(r, \boldsymbol{B}_{l}, f\right)+S(r, f) .
$$

Proof. We suppose without loss of generality that $q \geqq n+1$. Put for $j=$ $1, \ldots, q$

$$
\boldsymbol{A}_{\jmath}=\left[a_{j 1}, \ldots, a_{\jmath^{n+1}}\right], \quad \tilde{\boldsymbol{A}}_{\jmath}=\left(g_{j 1}, \ldots, g_{\jmath^{n+1}}\right), \quad\left(\boldsymbol{A}_{\jmath}, f\right)=F_{\jmath}
$$

and for $l=1, \ldots, \mu$

$$
\boldsymbol{B}_{l}=\left[a_{q+l 1}, \ldots, a_{q+l n+1}\right], \quad \tilde{\boldsymbol{B}}_{l}=\left(g_{q+l 1}, \ldots, g_{q+l n+1}\right), \quad\left(\boldsymbol{B}_{l}, f\right)=G_{l} .
$$

We may suppose without loss of generality that $\widetilde{\boldsymbol{B}}_{1}, \ldots, \widetilde{\boldsymbol{B}}_{\mu}, \boldsymbol{e}_{\mu+1}, \ldots, \boldsymbol{e}_{n}, \boldsymbol{e}_{n+1}$ are linearly independent over $\Gamma$ since $\widetilde{\boldsymbol{B}}_{1}, \ldots, \widetilde{\boldsymbol{B}}_{\mu}$ are linearly independent over $\Gamma$ and belong to $\tilde{X}(0)$.

For any integer $p$, let $V(p)$ be the vector space generated by

$$
\left\{\prod_{k=1}^{n+1} \prod_{j=1}^{q+\mu} g_{j k}^{p(\jmath, k)}: \sum_{k=1}^{n+1} \sum_{j=1}^{q+\mu} p(j, k) \leqq p, p(j, k) \geqq 0 \text { and integer }\right\}
$$

over $\boldsymbol{C}$ and

$$
d(p)=\operatorname{dim} V(p) .
$$

Then, $V(p)$ is a subspace of $V(p+1)$ and

$$
\liminf _{p \rightarrow \infty} d(p+1) / d(p)=1
$$

by the reduction to absurdity since $d(p) \leqq\left(\begin{array}{c}(n+1)(q+\mu)+p \\ p\end{array}\right)$ (see [5], [6]).

Let

$$
b_{1}, \ldots, b_{d(p)}, b_{d(p)+1}, \ldots, b_{d(p+1)}
$$

be a basis of $V(p+1)$ such that

$$
b_{1}, \ldots, b_{d(p)}
$$

form a basis of $V(p)$. Then, it is clear that the functions

$$
\left\{b_{t} f_{k}: t=1, \ldots, d(p+1) ; k=1, \ldots, n+1\right\}
$$

are linearly independent over $\boldsymbol{C}$. We put for convenience

$$
W=W\left(b_{1} f_{1}, b_{2} f_{1}, \ldots, b_{d(p+1)} f_{n+1}\right) .
$$


Note that $N(r, W)=S_{0}(r, f)$.

Let $z$ be a point of $\boldsymbol{C}-\{0\}$ where none of $\left\{\tilde{F}_{j}\right\}_{j=1}^{q_{j=1}}$ has poles. We rearrange $\left\{\tilde{F}_{j}(z)\right\}_{j=1}^{q_{j}}$ as follows :

$$
\left|\widetilde{F}_{J_{1}}(z)\right| \leqq \widetilde{F}_{J_{2}}(z)|\leqq \cdots \leqq| \widetilde{F}_{J_{n}}(z)|\leqq \cdots \leqq| \widetilde{F}_{\partial_{q}}(z) \mid,
$$

where $1 \leqq j_{1}, \ldots, j_{q} \leqq q$.

We have for $k \geqq n+1$

$$
\|f(z)\| \leqq K(z)\left|\widetilde{F}_{\jmath_{k}}(z)\right|
$$

and for $k=1, \ldots, q$

$$
\left|\widetilde{F}_{J_{k}}(z)\right| \leqq K(z)\|f(z)\|
$$

We then have the following from (9):

$$
\begin{aligned}
& \left(\prod_{j=1}^{q} \frac{\left\|\boldsymbol{A}_{j}(z)\right\|\|f(\boldsymbol{z})\|}{\left|\left(\boldsymbol{A}_{\jmath}, f\right)(z)\right|}\right)^{d(p)}=\left(\prod_{\jmath=1}^{q} \frac{\left\|\tilde{\boldsymbol{A}}_{j}(\boldsymbol{z})\right\|\|f(z)\|}{\left|\widetilde{F}_{j}(z)\right|}\right)^{d(p)} \\
& =\left(\prod_{j=1}^{q}\left\|\tilde{\boldsymbol{A}}_{j}(\boldsymbol{z})\right\|\right)^{d(p)}\left(\prod_{k=1}^{n} \frac{\|f(z)\|}{\left|\widetilde{F}_{\jmath_{k}}(z)\right|}\right)^{d(p)}\left(\prod_{k=n+1}^{q} \frac{\|f(z)\|}{\left|\widetilde{F}_{\jmath_{k}}(z)\right|}\right)^{d(p)} \\
& \leqq K(z)\left(\prod_{k=1}^{n} \frac{\|f(\boldsymbol{z})\|}{\left|\tilde{F}_{J_{k}}(z)\right|}\right)^{d(p)} .
\end{aligned}
$$

We note that by Lemma 3 , (a)

$$
\left|f_{n+1}(z)\right| \leqq K(z)\left\{\left|\widetilde{F}_{J_{k}}(z)\right|+u(z)\right\} \quad(k=1, \ldots, q) .
$$

since $a_{\jmath_{n+1}} \neq 0$ for any $\boldsymbol{A}_{j} \in X-X(0)$.

(I) The case when $u(z) \leqq\left|\widetilde{F}_{\jmath_{1}}(z)\right|$.

In this case we have from (12)

$$
\|f(z)\| \leqq K(z)\left|\widetilde{F}_{\partial_{k}}(z)\right| \quad(k=1, \ldots, n)
$$

and we have

$$
\left(\prod_{k=1}^{n} \frac{\|f(z)\|}{\left|\widetilde{F}_{\jmath_{k}}(z)\right|}\right)^{d(p)} \leqq K(z) .
$$

(II) The case when $\left|\widetilde{F}_{j_{1}}(z)\right|<u(z)$.

In this case we have from (12) for $k=1$

$$
\|f(z)\| \leqq K(z) u(z)
$$

and we have for $\Pi_{\mu}=\left|G_{1} \cdots G_{\mu}\right|$ and $\tilde{\Pi}_{\mu}=\left|\tilde{G}_{1} \cdots \tilde{G}_{\mu}\right|(\mu \geqq 1), \Pi_{0}=\tilde{\Pi}_{0}=1$ 


$$
\begin{aligned}
\left(\prod_{k=1}^{n} \frac{\|f(z)\|}{\left|\widetilde{F}_{j_{k}}(z)\right|}\right)^{d(p)} & \leqq K(z) \frac{u(z)^{(n+1) d(p)}}{\left(\Pi_{k=1}^{n+1}\left|\tilde{F}_{\jmath_{k}}(z)\right|\right)^{d(p)}} \\
& =K(z) \frac{\left(\tilde{\Pi}_{\mu}(z) u(z)^{n+1}\right)^{d(p)}}{\left(\tilde{\Pi}_{\mu}(z) \Pi_{k=1}^{n+1}\left|\widetilde{F}_{J_{k}}(z)\right|\right)^{d(p)}}=(*)
\end{aligned}
$$

by (10). Here, we put

$$
\tilde{G}_{\imath}=\tilde{H}_{\imath}(i=1, \ldots, \mu), \quad \tilde{F}_{j_{k}}=\tilde{H}_{\mu+k}(k=1, \ldots, n+1)
$$

and let

$$
\left|\tilde{H}_{\imath_{1}}(z)\right| \leqq\left|\tilde{H}_{\imath_{2}}(z)\right| \leqq \cdots \leqq\left|\tilde{H}_{\imath_{n+1}+\mu}(z)\right| .
$$

Then, for $k=2, \ldots, \mu+1$

and we have

$$
u(z) \leqq K(z)\left|\tilde{H}_{\imath_{n+k}}(z)\right|
$$

$$
\begin{aligned}
(*) \leqq & K(z) \frac{\left\{\tilde{\Pi}_{\mu}(z) u(z)^{n+1-\mu}\right\}^{d(p)}\left\{\Pi_{k=n+2}^{n+\mu+1} K(z)\left|\widetilde{H}_{\imath_{k}}(z)\right|\right\}^{d(p)}}{\Pi_{k=1}^{n+1+\mu}\left|\widetilde{H}_{\imath_{k}}(z)\right|^{d(p)}} \\
& \leqq K(z) \frac{\left\{\tilde{\Pi}_{\mu}(z) u(z)^{n+1-\mu}\right\}^{d(p)}}{\Pi_{k=1}^{n+1}\left|\widetilde{H}_{\imath_{k}}(z)\right|^{d(p)}} .
\end{aligned}
$$
that

Now $\tilde{H}_{i_{1}}, \ldots, \tilde{H}_{\imath_{n+1}}$ are linearly independent over $\Gamma$ and it is easy to see

$$
\left\{b_{1} \tilde{H}_{\imath_{1}}, b_{2} \tilde{H}_{\imath_{1}}, \ldots, b_{d(p)} \tilde{H}_{\imath_{n+1}}\right\}
$$

are linearly independent over $\boldsymbol{C}$. Since $\tilde{H}_{\jmath}=\left(\tilde{\boldsymbol{A}}_{\jmath}, f\right)$ or $\widetilde{H}_{\jmath}=\left(\tilde{\boldsymbol{B}}_{\jmath}, f\right)$, these functions can be represented as linear combinations of

$$
\left\{b_{t} f_{k}: 1 \leqq t \leqq d(p+1), 1 \leqq k \leqq n+1\right\}
$$

with constant coefficients :

$$
\left(b_{1} \tilde{H}_{\imath_{1}}, b_{2} \widetilde{H}_{\imath_{1}}, \ldots, b_{d(p)} \tilde{H}_{\imath_{n+1}}\right)=\left(b_{1} f_{1}, b_{2} f_{1}, \ldots, b_{d(p+1)} f_{n+1}\right) D_{1}
$$

where $D_{1}$ is an $(n+1) d(p+1) \times(n+1) d(p)$ matrix whose elements are constants. The rank of $D_{1}$ is equal to $(n+1) d(p)$. Let $D_{2}$ be an $(n+1) d(p+1) \times(n+1)$ $\{d(p+1)-d(p)\}$ matrix consisting of constant elements such that the $(n+1)$ $d(p+1) \times(n+1) d(p+1)$ matrix

$$
D=\left[D_{1} D_{2}\right]
$$

is regular. Put

$$
\left(K_{1}, \ldots, K_{L}\right)=\left(b_{1} f_{1}, b_{2} f_{1}, \ldots, b_{d(p+1)} f_{n+1}\right) D_{2},
$$

where $L=(n+1)\{d(p+1)-d(p)\}$, then

$$
\left(b_{1} \widetilde{H}_{\imath_{1}}, \ldots, b_{d(p)} \tilde{H}_{\imath_{n+1}}, K_{1}, \ldots, K_{L}\right)=\left(b_{1} f_{1}, \ldots, b_{d(p+1)} f_{n+1}\right) D,
$$


from which we obtain

$$
W\left(b_{1} \tilde{H}_{\imath_{1}}, \ldots, K_{1}, \ldots, K_{L}\right)=(\operatorname{det} D) W,
$$

where $W=W\left(b_{1} f_{1}, \ldots, b_{d(p+1)} f_{n+1}\right)$. We put

$$
W\left(b_{1} \widetilde{H}_{\imath_{1}}, \ldots, K_{1}, \ldots, K_{L}\right)=W\left(\jmath_{1}, \ldots, \jmath_{n+1}\right)
$$

as $\widetilde{H}_{i_{1}}, \ldots, \widetilde{H}_{\imath_{n+1}}$ are determined after $\left(j_{1}, \ldots, j_{n+1}\right)$.

We then have from (15)

$$
\begin{aligned}
\frac{1}{\left\{\Pi_{k=1}^{n+1}\left|\widetilde{H}_{\imath_{k}}(z)\right|\right\}^{d(p)}} & =\frac{\left|W\left(j_{1}, \ldots, j_{n+1}\right)(z)\right|}{|W(z)||\operatorname{det} D|} \cdot \frac{1}{\left\{\Pi_{k=1}^{n+1}\left|\widetilde{H}_{\imath_{k}}(z)\right|\right\}^{d(p)}} \\
& =\frac{1}{|\operatorname{det} D|} \cdot \frac{1}{|W(z)|} \cdot \frac{\left|W\left(j_{1}, \ldots, j_{n+1}\right)(z)\right|}{\left\{\Pi_{k=1}^{n+1}\left|\widetilde{H}_{\iota_{k}}(z)\right|\right\}^{d(p)}} \\
& \leqq K(z) \frac{\{u(z)\}^{L}}{|W(z)|} \cdot \frac{\left|W\left(j_{1}, \ldots, j_{n+1}\right)(z)\right|}{\left|b_{1} \widetilde{H}_{\imath_{1}}(z) \cdot b_{2} \widetilde{H}_{\imath_{1}}(z) \cdots K_{L}(z)\right|}
\end{aligned}
$$

since $\left|K_{j}(z)\right| \leqq K(z)\|f(z)\|(j=1, \ldots, L)$ and $\|f(z)\| \leqq K(z) u(z)$ in this case.

Further, by using the following inequalities for $j=1, \ldots, L$

$$
T\left(r, K_{j} / b_{1} \tilde{H}_{\imath_{1}}\right) \leqq 2 n T(r, f)+S_{0}(r, f),
$$

which we can prove as in Lemma 4 since $b_{t} \in \Gamma(1 \leqq t \leqq d(p+1))$, and by Lemma 4 , we have

$$
\frac{1}{2 \pi} \int_{0}^{2 \pi} \log ^{+} \frac{\left|W\left(j_{1}, \ldots, j_{n+1}\right)\left(r e^{i \theta}\right)\right|}{\left|b_{1} \widetilde{H}_{\imath_{1}}\left(r e^{i \theta}\right) \cdots K_{L}\left(r e^{i \theta}\right)\right|} d \theta=S(r, f)
$$

as usual (see [1], p. 12-p. 15).

From (11), (13), (14), (14') and (16), we obtain

$$
\begin{aligned}
d(p) \sum_{j=1}^{q} \log \frac{\left\|\boldsymbol{A}_{j}(z)\right\|\|f(z)\|}{\left|\left(\boldsymbol{A}_{\jmath}, f\right)(z)\right|} \leqq & \log ^{+} \frac{\tilde{\Pi}_{\mu}(z)^{d(p)} u(z)^{(n+1) d(p+\ddot{1})-\mu d(p)}}{|W(z)|} \\
& +\log ^{+} K(z)+\sum_{\left(j_{1}, \ldots, j_{n+1}\right)} \log ^{+} \frac{\left|W\left(j_{1}, \ldots, j_{n+1}\right)(z)\right|}{\left|\widetilde{b}_{1} \widetilde{H}_{\imath_{1}}(z) \cdots K_{L}(z)\right|}
\end{aligned}
$$

where $\sum\left(\jmath_{1}, \ldots, j_{n+1}\right)$ is the summation taken over all combinations $\left(j_{1}, \ldots, j_{n+1}\right)$ chosen from $\{1, \ldots, q\}$ which appear in the above argument when we vary $z$ in $0<|z|<\infty$.

Integrating both sides of (18) with respect to $\theta$ from 0 to $2 \pi\left(z=r e^{i \theta}\right)$, we obtain the inequality

$$
\begin{aligned}
d(p) \sum_{j=1}^{q} m\left(r, \boldsymbol{A}_{\jmath}, f\right) \leqq & \frac{1}{2 \pi} \int_{0}^{2 \pi} \log ^{+} \frac{\tilde{\Pi}_{\mu}\left(r e^{i \theta}\right)^{d(p)} u\left(r e^{i \theta}\right)^{\alpha(p)}}{\left|W\left(r e^{i \theta}\right)\right|} d \theta \\
& +S_{0}(r, f)+S(r, f)
\end{aligned}
$$


by (17), where $\alpha(p)=(n+1) d(p+1)-\mu d(p)$. Here,

$$
\begin{aligned}
& \frac{1}{2 \pi} \int_{0}^{2 \pi} \log +\frac{\tilde{\Pi}_{\mu}\left(r e^{i \theta}\right)^{d(p)} u\left(r e^{i \theta}\right)^{\alpha(p)}}{\left|W\left(r e^{i \theta}\right)\right|} d \theta \\
& \leqq \frac{1}{2 \pi} \int_{0}^{2 \pi} \log \left\{\tilde{\Pi}_{\mu}{ }^{2 d(p)}\left(\left|f_{1}\right|^{2 \alpha(p)}+\cdots+\left|f_{n}\right|^{2 \alpha(p)}\right)+|W|^{2}\right\}^{1 / 2}\left(r e^{i \theta}\right) d \theta \\
& -\frac{1}{2 \pi} \int_{0}^{2 \pi} \log \left|W\left(r e^{i \theta}\right)\right| d \theta+O(1)
\end{aligned}
$$

and as in the proof of Lemma 1 (when $\mu=0$ ) or Lemma 2 (when $\mu>0$ )

$$
\begin{gathered}
\frac{1}{2 \pi} \int_{0}^{2 \pi} \log \left(\tilde{\Pi}_{\mu}{ }^{2 d(p)}\left(\left|f_{1}\right|^{2 \alpha(p)}+\cdots+\left|f_{n}\right|^{2 \alpha(p)}\right)+|W|^{2}\right\}^{1 / 2}\left(r e^{i \theta}\right) d \theta \\
\leqq \\
\{(n+1) d(p+1)-n d(p)\} T(r, f)+(n-\mu) d(p) t(r, f) \\
+d(p) \sum_{l=1}^{\mu} N\left(r, \boldsymbol{B}_{l}, f\right)+S_{0}(r, f)+S(r, f) .
\end{gathered}
$$

For any positive number $\varepsilon$, let $p$ be so large that

$$
d(p+1) / d(p)<1+\varepsilon /(n+1)
$$

by (8). Then, from (19), (20) and (21) we obtain

$$
\sum_{j=1}^{q} m\left(r, \boldsymbol{A}_{\jmath}, f\right) \leqq(1+\varepsilon) T(r, f)+(n-\mu) t(r, f)+\sum_{l=1}^{\mu} N\left(r, \boldsymbol{B}_{l}, f\right)+S(r, f)
$$

since $N(r, W)=S_{0}(r, f)$.

As direct consequences of this theorem we have the followings as in Theorem 2, Corollaries 1 and 2.

COROLLARY 3 (Defect relation). Under the same assumption as in Theorem 3, we have

$$
\sum_{\jmath=1}^{q} \delta\left(A_{\jmath}, f\right)+\sum_{l=1}^{\mu} \delta\left(B_{l}, f\right) \leqq \mu+1+(n-\mu) \Omega .
$$

CoROllary 4. Let $X$ be a ע-maximal subset of $\boldsymbol{S}_{0}(f)$ and $\varepsilon$ be any positive number. Then,

(I) For any $A_{1}, \ldots, A_{q}$ in $X$

$$
\sum_{j=1}^{q} m\left(r, \boldsymbol{A}_{\jmath}, f\right) \leqq(\nu+1+\varepsilon) T(r, f)+(n-\nu) t(r, f)+S(r, f) .
$$

(II) $\quad \sum_{A \in X} \delta(A, f) \leqq \nu+1+(n-\nu) \Omega$.

Remark 4. $\nu+1+(n-\nu) \Omega \leqq n+1$ and the equality holds if and only if $\Omega=1$ or $\nu=n$. 
Acknowledgement. The author thanks the referee for valuable comments to improve the paper.

\section{REFERENCES}

[1] H. CAR'TAN, Sur les zéros des combinaisons linéaires de $p$ fonctions holomorphes données, Mathematica, 7 (1933), 5-31.

[2] H. Fujimoto, Value Distribution Theory of the Gauss Map of Minimal Surfaces in $\boldsymbol{R}^{m}$, Aspects Math., E21, Friedr. Vieweg \& Sohn, Braunschweig, 1993.

[3] W.K. Hayman, Meromorphic Functions, Clarendon Press, Oxford, 1964.

[4] M. Ru AND W. STOLL, The second main theorem for moving targets, J. Geom. Anal., I (1991), 99-138.

[5] N. StEInmetz, Eine Verallgemeinerung des zweiten Nevanlinnaschen Hauptsatzes, J. Reine Angew. Math., 368 (1986), 134-141.

[6] W. Stoll, An extension of the theorem of Steinmetz-Nevanlinna to holomorphic curves, Math. Ann., 282 (1988), 185-222.

[7] N. Toda, An extension of the derivative of meromorphic functions to holomorphic curves, Proc. Japan Acad. Ser. A Math. Sci., 70 (1994), 159-163.

[8] N. TODA, On the order of holomorphic curves with maximal deficiency sum, Kodai Math. J., 18 (1995), 451-474.

[9] N. TodA, On holomorphic curves of infinite order with maximal deficiency sum, Bull. Nagoya Inst. Tech., 46 (1994), 175-186.

[10] N. Toda, On subsets of $\boldsymbol{C}^{n+1}$ in general position, Proc. Japan Acad. Ser. A Math. Sci., 72 (1996), 55-58.

[11] H. Weyl and F. J. Weyl, Meromorphic Functions and Analytic Curves, Ann. of Math. Stud., 12, Princeton Univ. Press, Prirceton, 1943.

Department of Mathematics

Nagoya InSTITUTE OF TECHNOLOGY

Gokiso, Show A-KU, NAgOYA 466

JAPAN 